\title{
Novel and Live Tracing Methodology for Power Cables and Transformers Based on Power Line Carrier Signals
}

\author{
Heejung Byun, Sugoog Shon, ${ }^{*}$ and Soomi Yang \\ Department of Information and Telecommunication, The University of Suwon, \\ Bongdam-eup, Hwasung City, Kyunggi-do 18323, South Korea.
}

(Received May 17, 2017; accepted March 14, 2018)

Keywords: synchronized client server, tracing, zero crossing, cables, power line carrier, identification

The three-phase, four-cable type of power distribution lines branch into multiple circuits from local pole- or pad-mounted transformers, which makes the topology of lines complicated. A challenging issue is how to trace cables and transformers in a complicated distribution system. The proposed idea is based on a power line carrier signal, which has certain propagation characteristics along the distribution system. This novel and live tracing methodology, called the synchronized client and server method, is introduced, designed, and implemented to trace cables and transformers in the distribution system. The most difficult part of cable tracing is to isolate the signals needed for tracking from a mixture of signals routed through transformers or loads and signals transmitted directly along a test line. The algorithms needed to isolate the signals have been developed and a zero crossing sensor module is designed to ensure that the remote client and server are in phase. Simulations have been carried out to verify the proposed system using a Simulink simulator. Experiments have been conducted to verify the theoretical concepts in a test bed and a commercial building. Finally, the theory has been proved at a resolution of $0.5 \mathrm{~ms}$ from comparisons between the simulations and the experiments.

\section{Introduction}

In the case of disasters such as fires and typhoons or of new service provisions for buildings or customers as shown in Fig. 1, an electrician on site should be able to correctly figure out electrical configurations such as cables, circuit breakers, and transformer connections as soon as possible. Confusion with respect to cables, phases, and transformers can cause disproportionate concentrations of loads, which can cause phase imbalances that can lead to power loss or power failure and, thus, to equipment failure due to excessive voltage regulation or reduction of useful life. The unmistakable identification of cables and phases is an absolute safety-related task. The wrong selection can result in fatal consequences for the operator and cause the loss of supply for the connected customers. ${ }^{(1)}$

Electrical distribution systems that are classified into medium- and low-voltage systems deliver electricity from substations to consumers. Distribution systems mainly consist of

*Corresponding author: e-mail: sshon@suwon.ac.kr http://dx.doi.org/10.18494/SAM.2018.1870 

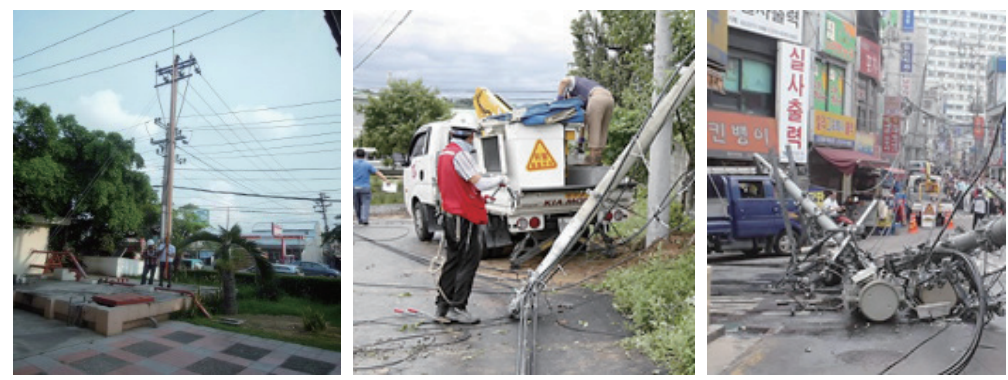

Fig. 1. (Color online) Places where electric circuit configuration must be identified.

cables, transformers, poles, breakers, and other components. Distribution topologies differ from country to country, and also within each country: for example, radial and interconnected distribution networks, delta and wye three-phase services, single-phase and split-phase lowvoltage services, and different earth systems. The three-phase, four-cable type with multiple grounds is the major type of distribution line in Korea. From medium voltage/low voltage (MV/ LV) transformers on an electric pole or pad, electric power with one or three phases is serviced through two, three, or four cables. Distribution lines classified into 'A', 'B', and ' $\mathrm{C}$ ' phases branch into multiple circuits to power local pole- or pad-mounted transformers. Therefore, as an electrician approaches the end of distribution lines, the identification of a phase becomes more difficult. Even worse, too many obstacles such as buildings, trees, or hills, make it harder to distinguish among phases.

The importance of phase identification, which is the process of measuring absolute phases for cables, was discussed previously. For phase identification, synchronization and comparison between the known reference phases at a substation and the unknown phase at a local site have been employed using GPS technology. Mutual synchronization has been accomplished by marking standard GPS time records to every phase value. ${ }^{(2-5)}$ However, phase identification supports neither transformer tracing, which identifies the transformer a customer is connected to, nor cable tracing. Accordingly, technology to simultaneously trace both cables and transformers needs to be developed.

A cable tracing or identification is used to distinguish cables serviced from local transformers or indoors where GPS signals are unavailable. Transformer tracing is used to figure out which transformer a customer is connected to. The cable and transformer tracing can be used as supplementary methods for phase identification.

For cable tracing, tone tracing is widely used, in which at one end of a dead cable one places an electrical signal using a tone generator, and then traces that signal, using a tone tracer. Unfortunately, it is simpler in theory than in practice, where some tones in electrical power cabling cannot discriminate phases. Another type of cable tracer uses a high-energy pulse to send a large impulse current signal from a pulse transmitter to a pulse detector that receives the signal. The problem is that the cable must be disconnected from any load impedance with the three-phase conductors tied together and grounded at the far end. In addition, another cable tracing that can single out a conductor or a cable among other conductors or cables has been developed on the basis of polarity impulse signals. With a flex coupler, only a single conductor 
or cable with the correct polarity can be detected amidst all other cables that have the opposite polarity. Another way to communicate coded messages between a central device and a line device through the distribution networks has also been developed. ${ }^{(6-8)}$

With the advent of power line carrier technology, methods for tracing transformers have been discussed, as seen in $\mathrm{Ku}$ et al., but their method cannot discriminate a specific cable among cables. ${ }^{(9,10)}$ As our previous work has established, ${ }^{(11)}$ transformer tracing is also possible. However, cable tracing is still challenging because there is a mix of signals originally sent from a server to a specific cable and the signals coming through loads or transformers. From the characteristics of power line communication (PLC) signal propagation along distribution lines, any signal sent by the server is directly and indirectly transferred to the client. Extraction of the proper signal from the mixed signals will only be possible with a fast signal processor, which can process within a few nanoseconds. ${ }^{(12)}$

As an extension of our previous work, we propose a novel and live synchronized client and server method, to trace both the cable and the transformer by adding a zero crossing sensing module and developing a time delay model. In the next section, the theory and algorithm for the tracing method is discussed. Then, a cable and transformer identification system is designed and implemented. The system consists of a server and a client. In tracing, the client and the server, both operating independently, are synchronized to distinguish the cable without using GPS at the moment it senses the zero crossing of the phase voltage. Experiments have been conducted to verify the theoretical concepts. Simulations have been carried out to understand the proposed system with newly built Simulink models. Finally, the results of experiments and simulations are compared to verify the proposed method.

\section{Synchronized Client and Server Method for Tracing Cables and Transformers}

\subsection{Theoretical considerations}

Let us consider a test customer as in Fig. 2. The test site on phase 'B' wants to know which transformer (Tr.1 or Tr.2) it is directly connected to. We need to determine whether the test customer is directly connected to Tr.2 or Tr.1. Obviously, if the distance between the client at

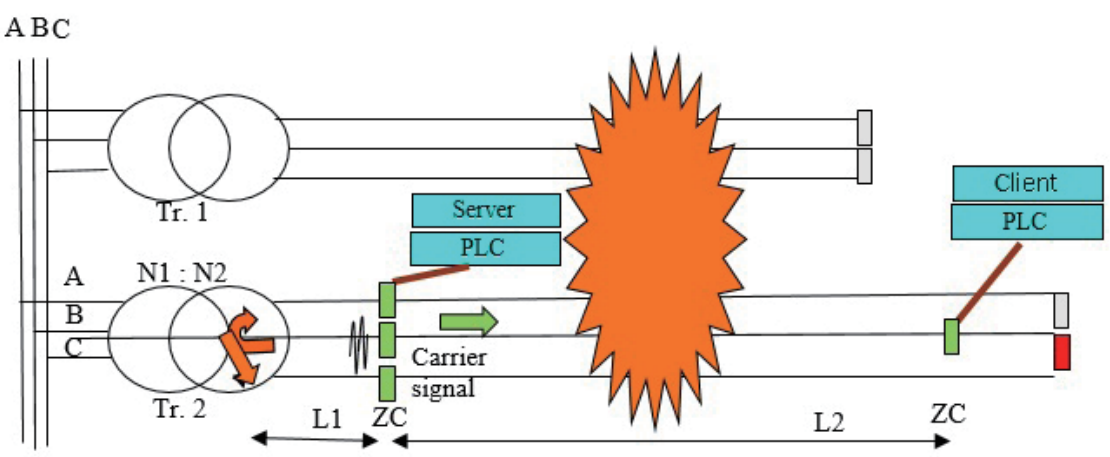

Fig. 2. (Color online) Concept of the synchronized client and server method. 
the test site and the server is close enough, we can successfully figure out the connectivity with an instrument like an oscilloscope. However, it is not easy to determine site-to-transformer connection if the test site and the server are far apart. Even with commercial phase finders, we do not know which transformer the test site is connected to because a phase finder does not reveal its connectivity to a transformer.

We propose a new method, called the synchronized client and server method, which reveals the cable connectivity between the client and the server with power line carrier technology. This method needs neither a fast microprocessor nor uses phase finders. If the client and the server are located on the same cable, they have the same phase of ' $\mathrm{B}$ '. Otherwise, they are assumed to have different phases such as 'A' or ' $C$ '.

The key challenge of this research is to determine if two sites at a distance from each other are on the same phase. If the client and the server include an internal clock with standard or absolute time, synchronization is easy. Realistically, that is beyond our scope. In previous studies, the client and the server operated independently with an independent internal clock. Accordingly, it is not easy to see that the client and the server are in the same phase. For synchronization between the client and the server, the event time when zero crossing has occurred is utilized as the reference synchronizing time. In alternating currents, the zero crossing is a transient point at which there is no voltage present. We use a zero crossing sensing module for detecting the point where the voltage crosses zero in either direction. The zero crossing sensing module indicates the phase start point of a voltage. The zero crossing time is used as a reference point for the determination of synchronization between the remote client and the server. We can say that the transmission distance of 'L2' is negligible because the propagation of the wave along the line is at about the speed of light.

In three-phase distribution lines, the server has three zero crossing points for synchronization markers differing by $120^{\circ}$ and the client will have a zero crossing point during every cycle as shown in Fig. 3. Assume that a server sends a message through the ' $\mathrm{C}$ ' phase line after zero crossing, as shown in the upper part of Fig. 3. The PLC signals injected into the ' $\mathrm{C}$ ' phase are divided into signals that are directly transmitted to the ' $\mathrm{C}$ ' phase line and signals that reach the 'A' phase or 'B' phase line through loads or transformers. Let $T_{\text {delay }}$ be the total transmission delay time it took the client to start receiving the message since the server started sending the message after zero crossing. A new term, synchronized phase discrimination time, $T_{s y n}$, is

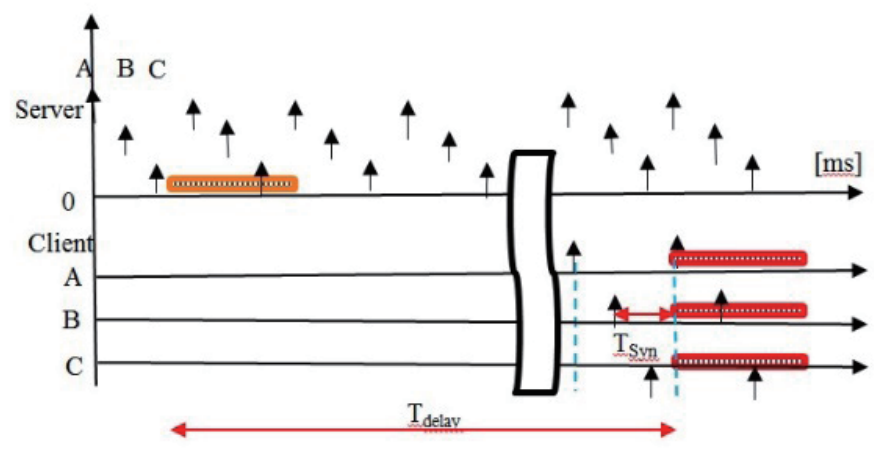

Fig. 3. (Color online) Synchronization and message transfer. 
introduced as a unit determining whether two sites are on the same phase or not; it is defined as the time elapsed between the last zero crossing and the beginning of the message received by the client. $T_{s y n}$ is measured differently depening on which phase is measured.

\subsection{Time delay model and analysis}

The communication process for the client and the server can be described as a layered structure like open system interconnection (OSI)'s seven layers. As in Fig. 4, the server is modeled with four layers and the client is modeled with three layers. When a zero crossing event occurs from one of the cables, the event causes a direct interruption of the server because the delay $T 1$ happens in microseconds. A message generated by the server upon the event is sequentially forwarded through the network with a delay of $T 2$, a data link delay of $T 3$, and a physical layer delay of $T 4$. Then, the message arrives at the client after a transmission delay of $T 5$ and is received by the client with a synchronized phase discrimination time of $T_{s y n}$ upon the last zero crossing. From the last zero crossing event by the client, the client receives and processes the message with a physical layer delay of $T 8$ and a data link layer delay of $T 7$. We define the total transmission delay, $T_{\text {delay }}$, as the time interval between the first zero crossing time by the server and the initiation of message received by the client. Its value is shown in Eq. (1).

$$
T_{\text {delay }}=T 1+T 2+T 3+T 4+T 5+T 6
$$

According to the new measured time, $T_{s y n}$, the phase of the cables is automatically determined. When $T_{s y n}$ is at a minimum, both sites are in the same phase. For example, if the client and the server are located on the same cable with phase ' $C$ ', the message is transferred after the synchronized phase discrimination time of $T_{\text {syn }}$ of $3.0 \mathrm{~ms}$. Otherwise, the message is transferred after the delayed time corresponding to $120^{\circ}$ (or $3.0+5.5 \mathrm{~ms}$ ) or $240^{\circ}$ (or $3.0+$ $11.1 \mathrm{~ms}$ ) for either the 'B' or 'A' phase, respectively. These results will be explained in the next sections.

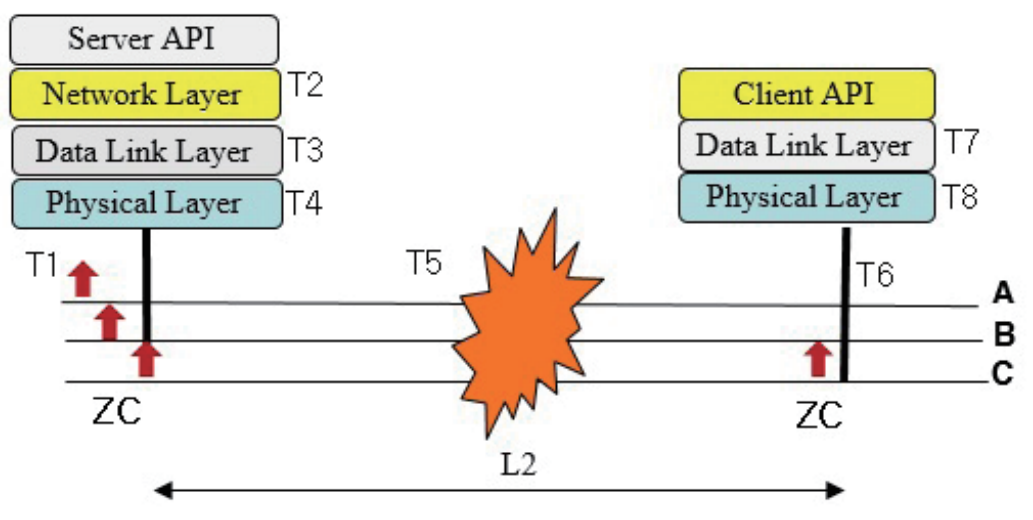

Fig. 4. (Color online) Time delay model. 


\section{Simulation Model and Analyses}

\subsection{Simulation model}

A simulation model for the synchronized client and server system has been studied with MATLAB Simulink software. For new simulation models, both a distribution system model and a communication delay model should be considered.

In this paper, distribution lines that have three-phase, four-wire multiple conductors with a multi-grounded neutral are modeled as a simple model shown in Fig. 5. The three-phase distribution model can include three-phase power sources, circuit breakers, transformers, loads, or the ground. The distribution lines can be modeled as a distributed parameter circuit based on transmitted wave theory rather than as a static model based on the circuit theory of Kirchhoff. ${ }^{(13)}$ The voltage source waveform applied to the lines is assumed to be a sinusoidal waveform. The individual phases of a three-phase voltage signal are $120^{\circ}$ apart, each with a frequency of 60 $\mathrm{Hz}$, and the symbols $\mathrm{A}, \mathrm{B}$, and $\mathrm{C}$ are assigned to each phase. Inside the simulation model, the $4 \times 4$ phase matrix consisting of four-wire grounded neutral line circuits is converted into a 3 $\times 3$ phase matrix consisting of the self and mutual equivalent impedances for the three phases with the "Kron" reduction method. ${ }^{(14)}$ Then, simulation equations of three sequence voltages are used, where the input parameters for the distribution lines are expressed by the sequence impedances of the line such that $Z_{00}=$ zero sequence impedance, $Z_{11}=$ positive sequence impedance, and $Z_{22}=$ negative sequence impedance.

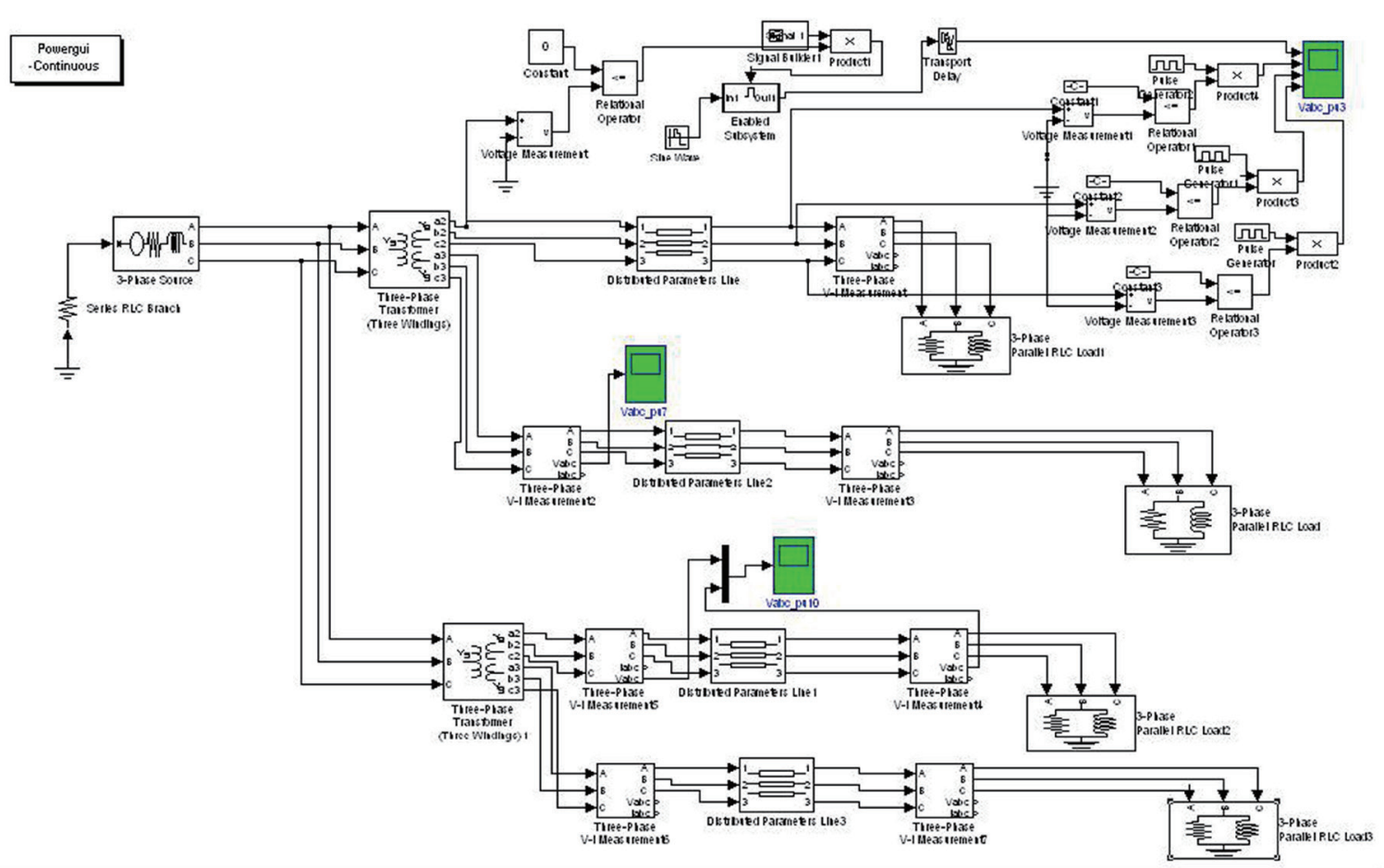

Fig. 5. (Color online) Simulink model for the synchronized client and server method. 
More details for the Simulink model, such as unbalanced loads, transformers, and grounding resister as well as zero crossing and a communication delay system, are explained as follows. First, the input source is expressed in terms of power with an internal resistance and an inductance. Power capacity that can provide sufficient power for use in the load is arranged. The neutral point is connected to the grounded resistance. The power output is provided to the distribution lines through a transformer wired as wye $(Y)$ or delta $(\Delta)$.

For the Simulink model of loads, various forms, such as constant impedance, constant current load, or constant capacity, can be used. A constant impedance model is a linear load that has a series of resistance, inductance, and capacitance values at a prescribed frequency. The effective and reactive power dissipated in the load has the characteristics of being proportional to the square of the applied voltage. The Simulink model of a constant current load is useful for allotting any current by lines. When modeling a transformer, the ratio between the primary and secondary voltages and the wiring method can be set. The transformers used in the simulation are composed of three-phase transformers, where wye $(Y)$ and delta $(\Delta)$ wirings are possible for the primary and secondary voltages. A transformer model that considers the internal loss generated by the internal resistance and the inductance is used. Recently, developed transformers have very low resistance and voltage drop. Ground is modeled as having only a resistive component. Ground resistance is assumed to be $5 \Omega$, which is a standard value for a $22.9 \mathrm{kV}$ multiple-grounding system. The primary side of the transformer, which is high in voltage, is $22.9 \mathrm{kV}$, and the secondary side is $220 \mathrm{~V}$. The ratio between the primary and secondary voltages is about $100(=22.9 \mathrm{kV} / 220 \mathrm{~V})$. For the distribution lines, parameters are represented as symmetric (zero, positive, negative) components. The length of the distribution line, 'L', can be scalable, and $1000 \mathrm{~m}$ is used here. The component values used in the simulation are as follows. ${ }^{(11,15-16)}$

$$
\begin{aligned}
& \text { Zero phase: } R_{0}=0.23 \Omega / \mathrm{km}, L_{0}=5.478 \mathrm{mH} / \mathrm{km}, C_{0}=0.008 \mu \mathrm{F} / \mathrm{km} \\
& \text { Positive phase: } R_{1}=0.17 \Omega / \mathrm{km}, L_{1}=1.21 \mathrm{mH} / \mathrm{km}, C_{1}=0.00969 \mu \mathrm{F} / \mathrm{km}
\end{aligned}
$$

In Fig. 6, more details for communication models are shown, and they are combined with the distribution system for the simulation that models the synchronization between the client and the server. Zero crossing and a total transmission delay model are developed and applied. The client and the server are synchronized at every zero crossing on their cables. After a zero crossing, the server sends a message with high-frequency signals instead of a real message. After the total transmission delay, the message arrives at the client.

From the simulation results as shown in Fig. 7, the top graph shows that the signal arrives at the client with a $T_{\text {delay }}$ of $450 \mathrm{~ms}$; the graphs below show zero crossing events for 'A', 'B', and ' $\mathrm{C}$ ' phases from the top. From the simulation, we realize that there is quite a delay in the time difference, a third of a cycle during the elapsed times, $T_{s y n}$. In the case in which the client and the server are positioned on the same cable, the $T_{s y n}$, after the zero crossing interrupt, is calculated to be about $2.6 \mathrm{~ms}$; in other cases, the calculation takes about 8.1 and $13.7 \mathrm{~ms}$, depending on the phases. 


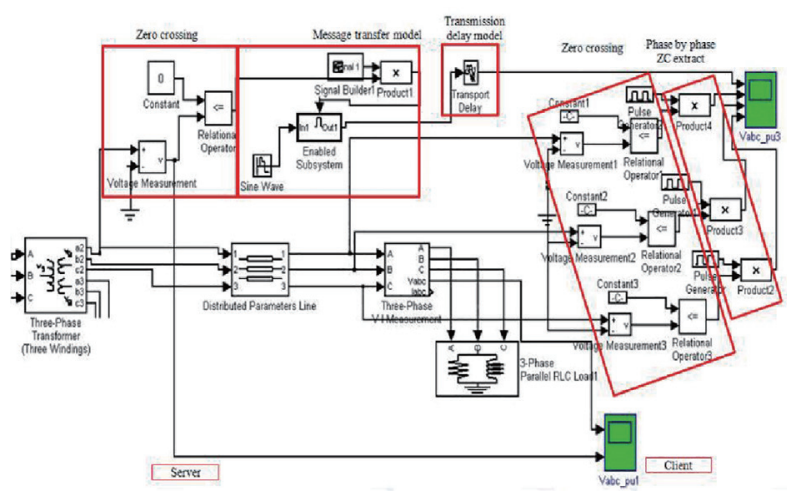

Fig. 6. (Color online) Synchronized simulation model.

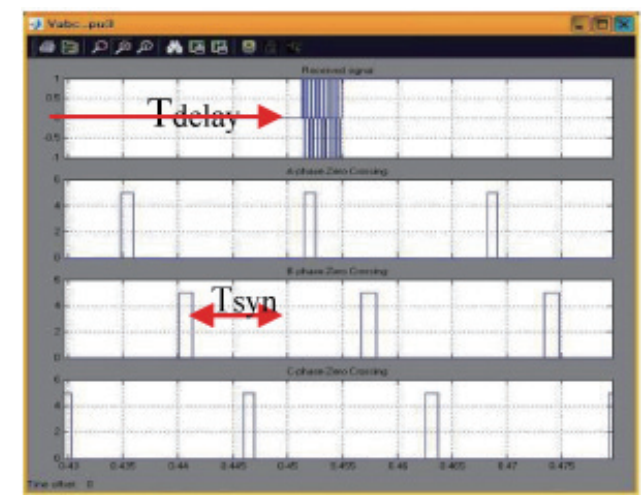

Fig. 7. (Color online) Simulated results.

\section{System Design and Algorithm}

Despite the growing use of digital communication systems, the PLC is still often the most cost-effective solution to cover the operational needs of a power network. The key advantage of the PLC is the use of existing electrical lines as a communication medium without the installation of additional networking infrastructures. Data is sent and received through communication channels by superposing modulated and demodulated high-frequency carrier signals on the power line voltage. PLCs have been used at all levels of the power network. A wide range of PLC technologies are needed for different applications such as telemetry, protection, and control by utility companies, along with metering and grid optimization by electricity providers. Besides these, advanced metering infrastructure (AMI) systems have recently become one of the most widely used applications. ${ }^{(17-19)}$

A test system to fulfill the proposed theory is shown in Fig. 8. The system is composed of a server and a client. The system is synchronized and operated at every zero crossing interruption by the zero crossing sensing module. Figure 9 shows the algorithm for the synchronized client and server method. The server sends a message upon a zero crossing interrupt and must wait for a time of at least $T_{\text {delay }}$ before sending the next message in order not to receive multiple messages by the client. The client starts a timer on every zero crossing event. When a message is received on any communication port of the client, then the time when the message arrives is recorded. Then, the time $T_{\text {syn }}$ is measured from the last zero crossing event. Finally, on the basis of $T_{s y n}$, we can determine whether or not the test site is situated on the same cable or the sender phase.

\section{Experiments and Results}

Typically, the distance between the server and the client is less than $1000 \mathrm{~m}$. To achieve a typical distribution line coverage, narrow-band PLC (NBPLC) is adopted for cable tracing instead of broadband PLC (BPLC), which has the transmission distance of only 200-300 m and data rate greater than $1 \mathrm{MHz}$. The NBPLC modem chip with a carrier frequency of 290 


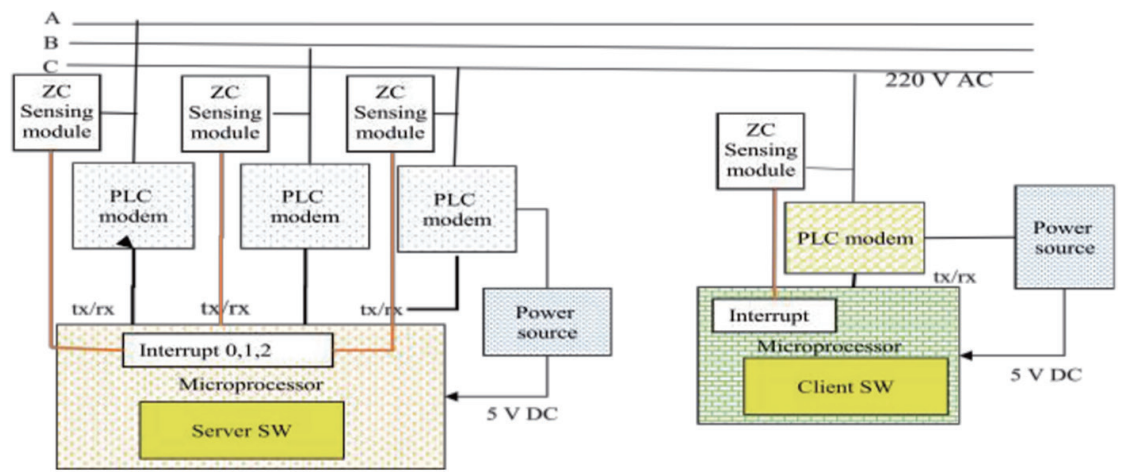

Fig. 8. (Color online) Design of synchronized client and server system.
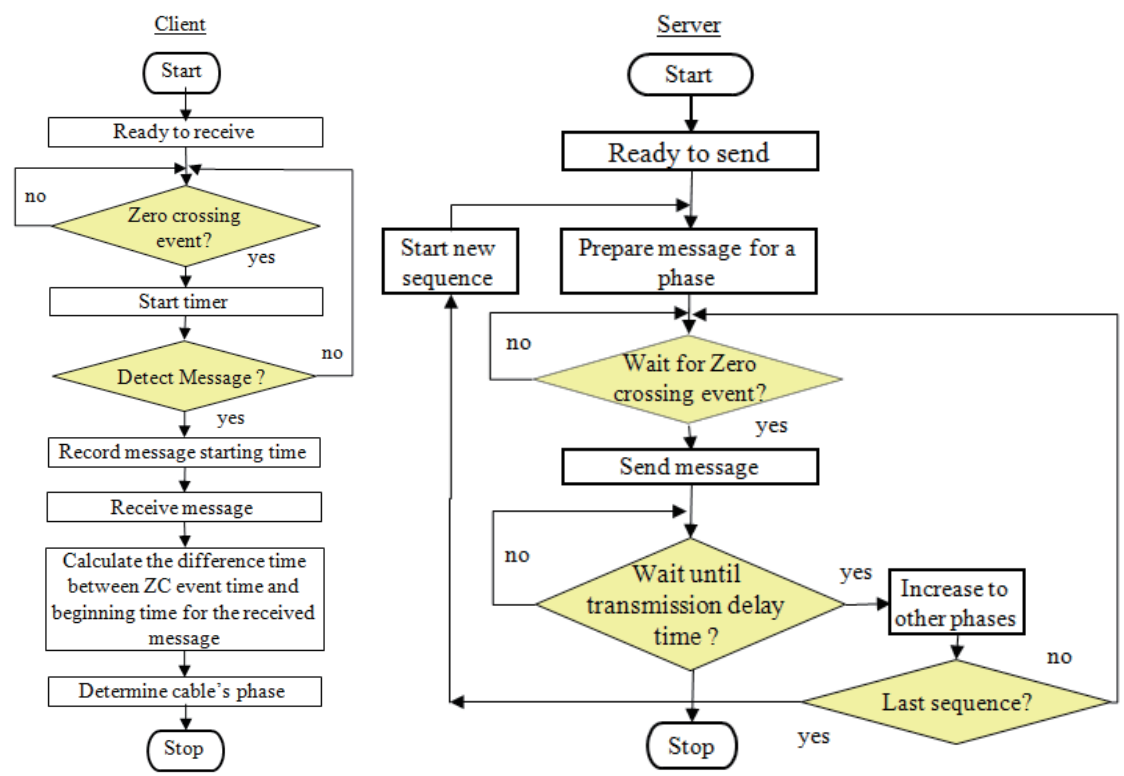

Fig. 9. (Color online) Algorithm for client and server.

$\mathrm{kHz} / 125 \mathrm{kHz}$, a bandwidth (BW) of the channel of $500 \mathrm{bps}$, and a simple medium access control (MAC) algorithm is used in this study. Arduino boards are also used as a common type of microprocessor. After implementing the proposed system, the experimental setup shown in Fig. 10 is constructed. The three-phase, four-wired voltage source can be controlled along with the phase angle of the source.

From some experiments, we observed that the server and the client were synchronized at the zero crossing event as in Fig. 11. Figure 12 shows a total $T_{\text {delay }}$ of about $450 \mathrm{~ms}$. Recall that the speed of universal serial communication ports is slow for Arduinos.

Several measurements for $T_{s y n}$ were made and shown in Fig. 13. After sending out a message from the ' $C$ ' phase of the server upon zero crossing, the client can receive the message on one of either 'A', 'B', or ' $\mathrm{C}$ ' phases after the $T_{\text {delay }}$ upon zero crossing. In these measurements, the 27 th zero crossing eventually becomes the last zero crossing, which becomes the starting point 


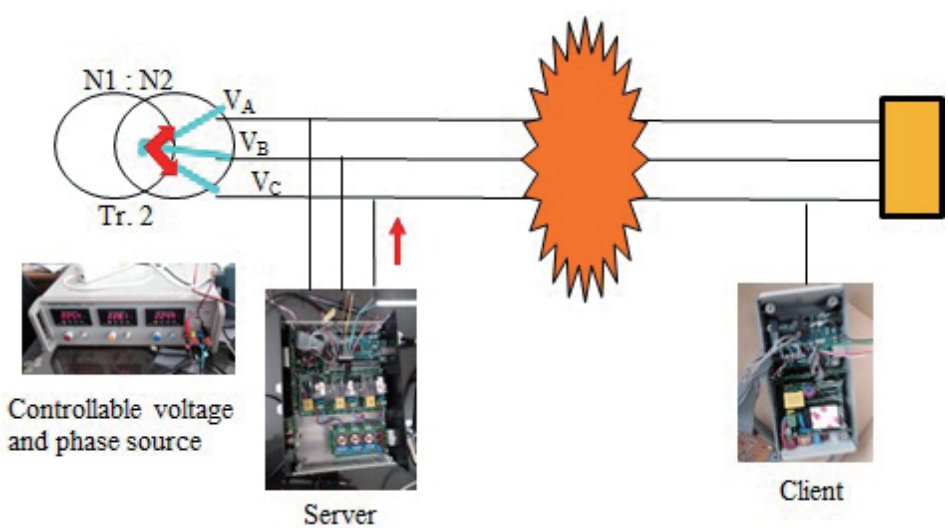

Fig. 10. (Color online) Experimental setup.

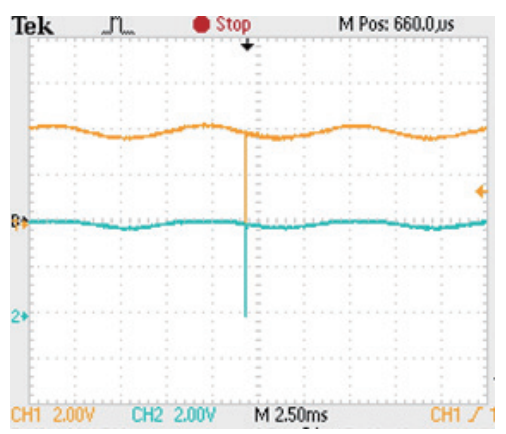

Fig. 11. (Color online) Zero crossing at the server (top) and the client (bottom).

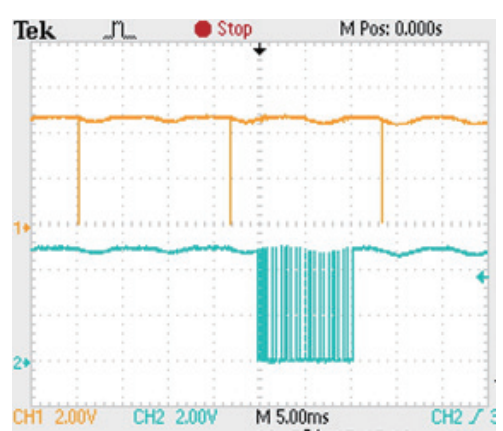

(a)

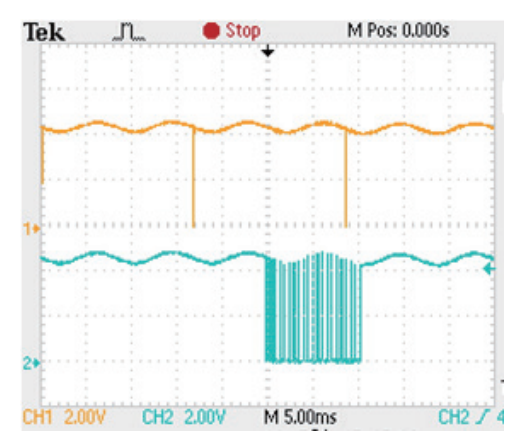

(b)

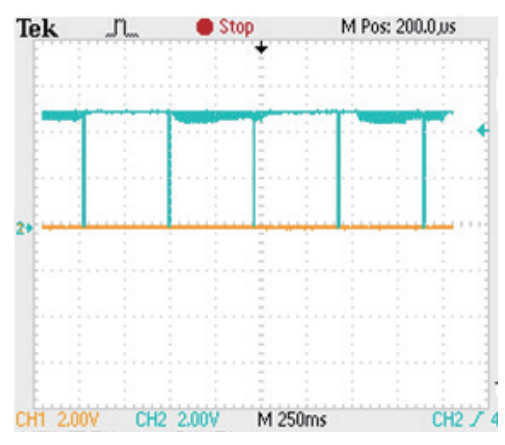

Fig. 12. (Color online) Total transmission delay $\left(T_{\text {delay }}\right)$.

Fig. 13. (Color online) $T_{\text {syn }}$ measurements for three different cases, measured of (a) 'A', (b) 'B', and (c) 'C' phases.

for $T_{s y n}$. A fixed length of 20 bytes is used for the message, which can include the management number of electric poles and the phase identification string. There is quite a difference among $T_{\text {syn }}$ values that enable us to tell whether the phase of the test client site is equal to the server phase or not. When the client and the server are positioned at the same cable, $T_{\text {syn }}$ is measured to be about $3 \mathrm{~ms}$, which is the smallest value among all results. 
Table 1

Comparisons between experiments and simulations.

\begin{tabular}{lccc}
\hline Server (phase) & Client (phase) & $T_{\text {syn }}(\mathrm{ms})$ (measured) & $T_{\text {syn }}(\mathrm{ms})$ (simulated) \\
\hline \multirow{3}{*}{$\mathrm{C}$} & $\mathrm{A}$ & $13.0-14.0$ & 13.7 \\
\cline { 2 - 4 } & $\mathrm{B}$ & $7.8-8.8$ & 8.1 \\
\cline { 2 - 4 } & $\mathrm{C}$ & $2.5-3.5$ & 2.6 \\
\hline
\end{tabular}

Table 1 shows the comparison between the measured $T_{s y n}$ and the simulated $T_{\text {syn }}$. The simulated and experimental results are highly consistent. Similar results were observed in repeated tests, unbalanced voltage tests using controllable voltage sources, and even in field experiments conducted inside an actual commercial building with distribution three-phase voltage. Similar results were also obtained even when communication ports for the server messages were changed into other phases. From the experiments, it can be concluded that the synchronized phase discrimination time, $T_{s y n}$, can be used as a reliable and accurate method to discriminate among cables.

\section{Conclusions}

A novel and live cable and transformer tracing method based on the synchronized client and server method has been proposed and implemented. The theoretical considerations and algorithms for discriminating the cables were introduced. New simulation models composed of both a distribution system model and a communication delay model have been introduced. Simulation models can calculate the time elapse of $T_{s y n}$ with Simulink. From the comparison between experiments and simulations, we conclude that the synchronized phase discrimination time, $T_{\text {syn }}$, can be used as an accurate and reliable measurement unit for the proposed method. The proposed method has also been verified in several experiments in an actual commercial building. Even though there may have been voltage imbalances or some disturbances that caused PLC modem characteristics, it seems that the method has a resolution of $\pm 0.5 \mathrm{~ms}$. In future work, we will determine the measurement coverage and improve the test distance.

\section{Acknowledgments}

This research was supported by the Basic Science Research Program through the National Research Foundation of Korea (NRF) funded by the Ministry of Science, ICT \& Future Planning (2107R1A2B1007779). The advice received from S. J. Choi of Editech, Ltd., is highly appreciated.

\section{References}

1 J. McDonald: 2nd, CRC Press (2007).

2 M. Bouvrette: US Patent No. 4626622 (1986).

3 L. A. Pomatto: US Patent No. 5510700 (1996).

4 G. H. Piesinger: US Patent No. 7031859 (2006).

5 H. J. Byun and S. G. Shon: J. Electr. Eng. Technol. 8 (2013) 742. 
6 Impulse Current: http://www.sebakmt.com/products/cable-identifier.html (accessed November 2017).

7 Flexible Coupler: http://www.powerpoint-engineering.com BAUR KSG 100 cable identifier (accessed November 2017).

8 Coded Messages: http://www.fluke.com/fluke/m2en/electrical-testers/electrical-testers/fluke-2042.htm (accessed November 2017).

9 C. S. Chen, T. T. Ku, and C. H. Lin: IEEE Trans. Ind. Appl. 46 (2010) 1072.

10 T. T. Ku, C. S. Chen, C. H. Lin, and M. S. Kang: The 4th IEEE Conf. Industrial Electronics and Applications (ICIEA, 2009) 3476-3481.

11 H. J. Byun and S. G. Shon: Int. J. Control Autom. Syst. 8 (2015) 63.

12 H. J. Byun and S. G. Shon: Int. Conf. Mechanics, Materials and Structural Engineering (Atlantis Press, ICMMSE 2016) 197.

13 W. H. Kersting: Distribution System Modeling and Analysis (CRC Press, Boca Raton, 2002).

14 G. Kron: AIEE Transactions Power Application Systems 71 (1952) 814.

15 F. L. Bertotti, M. S. Hara, and P. J. Abatti: Rev. Sci. Instrum. 81 (2010) 115106.

16 IEEE Recommended Practice for Grounding of Industrial and Commercial Power Systems, IEEE Std-1421991 (1992).

17 H. C. Ferreira, L. Lampe, J. Newbury, and T. G. Swart, Eds.: Power Line Communications: Theory and Applications for Narrowband and Broadband Communications over Power Lines (Wiley \& Sons, UK, 2010).

18 A. Cataliontti and G. Tine: 2009 XIX IMEKO World Congress (2009) 892.

19 T. Xie and G. Kim: J. Inf. Commun. Converg. Eng. 8 (2010) 258.

\section{About the Authors}

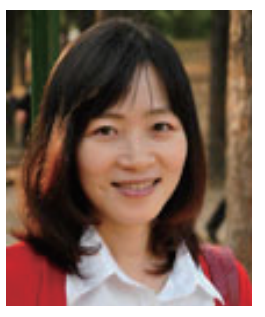

Heejung Byun received her B.S degree from Soongsil University, Korea, in 1999, an M.S. degree from Korea Advanced Institute of Science and Technology (KAIST), Korea, in 2001, and a Ph.D. degree from KAIST in 2005. She was a senior researcher in Samsung Electronics, Ltd. from 2007 to 2010. She is currently an assistant professor with the Department of Information and Telecommunications Engineering, the University of Suwon, Korea. Her research interests include network protocol, network modeling, controller design, and performance analysis.

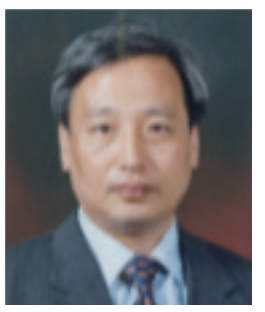

Sugoog Shon received his B.S. degree (1982) and his M.S. degree (1984) in Electrical Engineering from Seoul National University, and his Ph.D. degree (1996) in Electrical and Computer Engineering from the University of Texas at Austin. He is a professor in the Department of Information and Telecommunication at the University of Suwon in Korea. His research interests include computer and embedded systems, robots, network protocol, network simulation, and network programming.

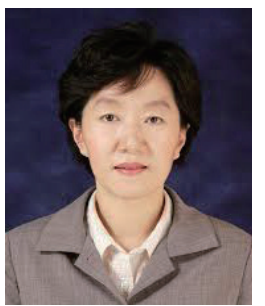

Soomi Yang received her B.S., M.S., and Ph.D. degrees in computer engineering from Seoul National University of Seoul, Korea, in 1985, 1987 and 1997, respectively. From 1988 to 2000, she was a researcher at Korea Telecom Research Center where she worked on telecommunication networks and internet and information security. From 2000 to 2001, she was a visiting scholar at UCLA, USA. From 2002 to 2004, she was a faculty member of Suwon Science College, Korea. She is currently an associate professor in the Department of Information Engineering, The University of Suwon, Korea. Her research interests include access control, network security, and secure system software. 